\title{
EMG Applications in Studies of Arts
}

\author{
Gongbing Shan and Peter Visentin \\ University of Lethbridge \\ Canada
}

\section{Introduction}

For researchers, surface electromyography (EMG) seems a seductive technology because it provides a non-invasive means to acquire in-vivo data about physiological processes that cause muscles to produce movement. It captures muscle activity during any movement of a body by measuring seizure potentials released by the muscles when they are activated. Consequently, measured potentials are typically used to fractionate a body's response process as well as get timing of neural control and coordination among muscle groups. Such analyses permit assessments of technical skills which, in turn, provide foundational information for biofeedback training and development of new pedagogical methods. Consequently, in high-skill activities such as those of the performing arts, EMG would appear to have significant relevance in terms of skill optimization and learning.

On the surface, it is apparent that arts performance shares many characteristics, including health risks, with an area where EMG research has already had a significant disciplinary impact - sports (Chesky, et al., 2002; Lehmann \& Davidson, 2002; Wilson, 1986). Pedagogy and training in both sports and performance arts aim to develop physical skills efficiently while avoiding injury for participants. While EMG applications in human sports movements have been an acknowledged aid in improving sport performance for decades (e.g. the Journal of Electromyography and Kinesiology has a 21-year history in this regard), applications in arts performances are still rare. Further, a review of the literature shows that, EMG applications in the arts tend to fall into only three broad categories: skill analysis descriptions and establishment of norms, training method evaluations, biofeedback in both pedagogy and injury remediation (Kjelland, 2000).

This book chapter details selected examples where EMG technology is used in applications pertinent to arts performance. Since almost all EMG studies rely upon initial skill analysis and description in order to establish norms, research in the performance arts is often a multi-stage process. Exemplars for purposes of discussion will be drawn from instrumental and vocal performance, as well as ballet. The goals of the selected research focus on: 1) describing the activity of selected muscles, 2) identifying innate abilities or talents, 3) quantifying the efficiency and effectiveness of pedagogical methods, 4) diagnosing technical difficulties, or 5) determining loading intensity during performance and evaluating factors related to pain and injury. Given the small body of existing EMG studies in the arts, the examples below are mainly chosen to elaborate the potential and relevance of EMG research in the performing arts as well as to encourage more research in the area. 


\section{Representative examples}

\subsection{Skill analysis}

In the areas of skill analysis and evaluation of training, EMG methods in music research are typically borrowed from kinesiology and sports, where they are used to quantify athletic potential and/or development, effectiveness of muscle group coordination and degree of motor control optimization (Riley \& Chong, 2010). To accomplish skill analysis, researchers' choice of data processing methods and analysis techniques play an important role. Since EMG signals are dependent on individual biological make-up (e.g. skin thickness, subcutaneous layers of fat or other soft tissues, etc.) and technical factors (e.g. placement of the sensors), any raw data gathered is unavoidably non-quantitative as a measure of neuralmuscular activity. For example, a sumo wrestler might generate lower measured EMG signals than a 90-pound ballerina, yet no one should be surprised at the outcome of a basho match-up between them. Thus raw data from EMG is typically processed in a number of ways. The technique of enveloping stabilizes signals for researchers and, in order to provide quasi-quantification, normalization of enveloped data is required (Kjelland, 2000; Morasky, Reynolds, \& Clarke, 1981; Zinn, 1998). By expressing all measurements as a percentage of a chosen reference value (i.e. normalization), EMG provides a viable means of comparison among different subjects and trials. Commonly used references for normalization include: 1) the maximum signal level during a trial, or 2) a Maximum Voluntary Contraction (MVC) determined in a separate trial under high-load conditions.

One particularly illuminating example of music skill analysis in the existing literature looks at the singing of onsets (Shan \& Visentin, 2010). "Onset" is a term used in music to describe the start of a pitch or tone. Whether singing or playing an instrument, onsets require a highly coordinated physical effort to generate sound. The articulatory nature of them is a vital component of musical expression. Thus, control of this element is important for all musicians. Since consistency and ease of effort during singing/playing can be taken as a measure of skill, analysis of this element can be equally important for researchers as an evaluative measure of skill development during training.

Figure 1 shows rectus abdominis activity of a trained mezzo-soprano singer executing onsets at three different pitch levels. Each trial consisted of ten onsets performed in two sets of five. Each set of five entailed singing a single pitch using four short vocalizations (approx. 1s. each) followed by one long vocalization (approx. 4s.). The singer was permitted to take a small breath between each of the onsets. There was about a two second break between each set of five. This same protocol was used at three different pitch frequencies - high (black), medium (dark grey) and low (light grey) covering the vocal range typical for a mezzo-soprano.

In terms of skill analysis, Figure 1 allows evaluation of training through an examination of the consistency and repeatability of the singing and the muscular activity used to accomplish the singing. Varying EMG amplitudes show that significantly different amounts of physical effort were required at different pitch levels. For low and medium pitch, although there was still a clear pattern of muscular activity, there was insignificant difference in EMG data between singing and rest with peak levels $10 \%-20 \%$ of the MVC. At high pitch, peak levels of EMG were above $60 \%$ of the MVC. Together these show a nonlinear relationship between pitch and effort required to sing. Importantly, at the highest pitch level there was a notable difference in physical effort between the beginning of the longer sustained tone and its end. Between onsets, low EMG measurements show disciplined anticipatory preparation typical of highly skilled individuals. Such results have 
implications for vocal pedagogy, since perceptual awareness of physiological phenomena allows coaches to direct training.

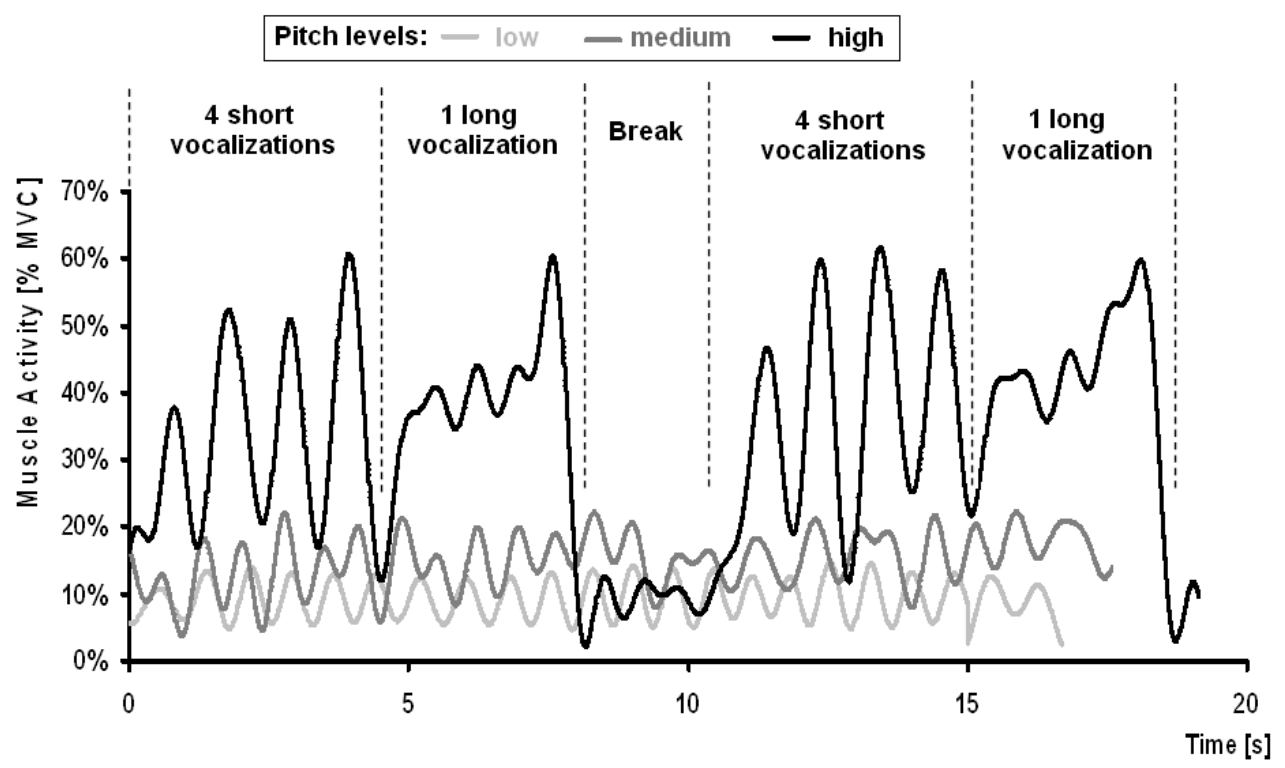

Fig. 1. Rectus abdominis activity of a trained singer at different pitch frequencies.

Evaluations of skills using EMG such as the one above can identify muscle recruitment strategies and provide information about fine motor training for music learners and professionals by showing physical adaptations resulting from training. In music, it is generally accepted among researchers and performers that that sustained levels of muscle contraction inhibit smooth motor movements and contribute to fatigue in both practicing and performing. EMG provides an excellent tool for measuring these characteristics, providing objective information that may help enhance artistic outcomes.

\subsection{Pedagogical applications}

A 1989 study broke new ground in the performing arts area when it used EMG to compare three different technical approaches to playing the piano (Bejjani et al., 1989). In a case study, a professional-level pianist was asked to perform three pianistic tasks using three defined postural orientations of the hand. Although it may seem to the reader somewhat artificial to have the same player adopt multiple postural orientations for the same musical passage, such adaptations are common when performing since the act of performance is by definition one of adaptation to "the moment". Rather than simply measuring a postural orientation to establish a "norm", the study validated the utility of EMG as a means to discriminate technique-related differential muscle activity. Where some muscle activity appeared to be posturally and task independent, others were clearly technique related. For the anterior deltoid, it was postulated that EMG activity during playing of arpeggios (one of the three tested tasks) was related primarily to idiosyncrasies of the performer since there 
was no significant difference in data among the three techniques examined. On the other hand, the flexor carpi radialis showed significant changes between techniques. Such findings have potential ramifications for pedagogy. Based on Bejjani's observations, since changes in technique do not alter the data, the deltoid muscle could effectively be neglected during training and the pedagogical focus can be directed toward those that muscles that are technique dependent. However, this conclusion might be misleading. The experiment design examined predetermined postural orientations of the hands and wrists - a three segment physics chain: hand, forearm, \& upper arm - essentially eliminating the significance of the trunk. In piano performance it is more and more understood that the trunk of the body plays a vital role, an examination of which would require a minimum of a four-segment chain. It may be the case that the deltoid is vital in some other way not examined in Bejjani's work. Far from being a criticism of Bejjani's study, this underscores his assertion that there is a great need for more varied EMG studies to be undertaken in the piano performance area.

Pedagogical potential of EMG research was further underscored in 1991 in a study by Heuser and McNitt-Gray. These researchers undertook skill analyses associated with trumpet embouchure and tone production (Heuser \& Mcnittgray, 1991). On the trumpet, quality of a tone's commencement is a result of careful synchronization of breath, lips and tongue. Hence from a muscular standpoint, the interaction of small and large muscles must occur in a very delicate balance. It is a fact that almost all players at one time or another can experience difficulties in achieving the effects they desire. Heuser's study examined embouchure muscles in preparation for playing both sustained and staccato (short) tones. Results indicated that EMG was an effective way to identify some aspects of tone production difficulties even for the very small muscles associated with the embouchure. In a later study, Iltis \& Givens undertook similar work focussed on comparing the EMG of a horn player exhibiting embouchure distonia with that of healthy performers (Iltis \& Givens, 2005). From a pedagogical standpoint such work could lead to the development of compensatory strategies for players who suffer from such vocational musculoskeletal disorders.

As in music, the use of EMG in dance can be highly varied. Its measurements can yield useful information about neuromusculoskeletal processes as well as artistic outcomes in performance. In spite of performance variability and the versatility of motor control among dancers, data has been used a means to distinguish experts from novices, track training changes and measure learning outcomes. Variability in data can illuminate temporal relationships between neural and musculoskeletal patterns with an ultimate goal of facilitating comparisons and developing normative data sets as reference points for training, performance, clinical practice, and research (Chatfield, 2003).

Studies such as the one by Chatfield (2003) show EMG to have significant pedagogical potential. For muscles that are truly technique independent, the training process can be simplified by paying more attention to other muscles. Further, idiosyncratic behaviours of a learner might be more easily observed in erratic or abnormal activity of such muscles. For muscles that are technique dependent, it is possible that either: 1) EMG patterns observed in high level professionals may be used as goals to train toward because they represent the most efficient and effective use of the body notwithstanding choice of technical approach, or 2) they can be used to evaluate the effectiveness of various training methods, and indeed develop new ones, by observing those that achieve the desired muscle activity effects most rapidly. 


\subsection{Vocational health and wellness applications}

In addition to its pedagogical utility, EMG has important medical applications relevant to arts performance. In the arts, as in sports, preventive maintenance toward long-term personal well-being is becoming more widely understood as a foundational goal of training, even though the underlying motivations for this ethical shift may differ between the two disciplines. In many sports, it has been generally understood that injuries are a part of the "game". However, since the real cost of a multi-million dollar athlete missing a game is very high, injury avoidance can be motivated by the economics of the sports business model. In the performance arts, economic necessity is not generally the primary driver of concerns in this regard for two reasons: 1) artists generally do what they do because they love it to the degree that "self" is subjugated to the artistic goal (both from health \& wellness and financial standpoints), and 2) the arts are seldom driven by a profit-oriented business model. Unfortunately, and perhaps as a consequence of the above, injury rates among performing artists are extraordinarily high but the investment in injury prevention is extremely low. Reported rates of musculoskeletal injuries in professional musicians range between $50 \%$ and 76\% (Fishbein, Middlestadt, Ottati, Straus, \& Ellis, 1988; Fry, 1986, 1988; Middlestadt \& Fishbein, 1989; Zaza, 1998).

Fortunately, conditions are currently ripe for more arts medicine research mainly because of a dogged dedication to raising awareness by organizations such as the Performing Arts Medical Association. In its nearly 30 years of efforts, it has become an inclusive alliance of the medical, therapeutic, scientific and artistic communities that deals with vocational issues of artists ranging from musculoskeletal disorders to psychological ones. As of the year 2010, there were at least 17 specialized centers in the United States that focus on performing arts medicine, whereas a decade ago there would have been only a small handful. The rising interest is proof that there is much more to be done, both in terms of treatment and prevention. EMG has the potential to be a fundamental tool in both injury prevention and remediation research.

Some of the most effective uses of EMG in this area have been in research that brings multiple technologies to bear on a single question. Multi-modal research can provide an excellent means of better understanding factors associated with vocational injuries (Visentin, Shan, \& Schultz, 2004). In a recent study of repetitive loading of the lower limb, patellar disorders, a condition common in dancers, was examined using EMG and magnetic resonance imaging MRI (Albisetti, Bartolomeo, Gabbiadini, Ventura, \& Parrini, 2008). The case study involving a professional ballet dancer suffering from anterior knee pain confirmed EMG as a useful method to help diagnose and guide treatment. The research was able to demonstrate the role of particular muscles in patellar maltracking, in this case by determining a difference in onset latency of the vastus medialis obliquus and vastus lateralis of the dancer's painful knee as compared to her asymptomatic one.

Other recent research has also made efforts to enhance the significance of EMG by using multi-modal technologies. It is now well accepted that postural and behavioural practices are factors that can influence the development of musculoskeletal movement disorders. In a study investigating causal factors underlying the aetiology of repetitive stress injuries in professional violinists, EMG was used in conjunction with 3-dimensional motion analysis technology and biomechanical modeling to find relationships between posture and internal loading intensity, laying the groundwork for developing injury prevention strategies (Visentin \& Shan, 2003; Visentin, et al., 2004). In these linked studies, EMG was used to determine loading patterns on the muscles and biomechanical modeling was used to 
confirm and further quantify the results. The left shoulder during violin playing proved to be static while right shoulder exhibited more varied behaviours ranging from quasi-static to dynamic. For the left arm, static shoulder loads were present regardless of the string being played or speed of the activity. Notably, the lowest level of the "dynamic" right shoulder was comparable to the level of the left shoulder. The maximum observed muscle intensity of the right side was four times higher than that of the left (a finding confirmed by both EMG and joint load analysis. Based on statistical studies, shoulder injury rates are comparable ( $51 \%$ for the right and $49 \%$ for the left) despite the differing kinematic and kinetic patterns (Fry, 1988). Through this kind of comparison the study demonstrated the significance of the type of muscle activity in terms injury causality. Looking at absolute values, the study showed that risk levels were essentially identical between static and dynamic loads even when dynamic loading was shown to be up to three or four times greater.

The implications of such research are far ranging and can influence pedagogy, learning, injury prevention and remediation. EMG can provide objective data that serves as a basis for understanding of biological and physiological process involved with arts performance. Further it can aid in evaluating and establishing pedagogical "best practices". For example, the results of the violin study above suggest the need for development of more refined teaching strategies and in a later study the same authors indeed do extrapolate from the data in developing such strategies (Visentin, Shan, \& Wasiak, 2008). Discussion in this article suggested some prevention strategies might be developed to include: 1) consciously invoking movements that minimize static postures (For example, to prevent left shoulder injuries, it appears a logical strategy to change the static nature of the muscle activity), 2) transfering loads from one muscle group to another (perhaps by using subtle postural changes that alternate the motor control pattern), or 3) reconceiving the playing mechanism in a variety of different ways to minimize unnecessary muscle loading.

It must be noted that applications of EMG in the performing arts may involve changing some of the conceptual paradigms of EMG research. In the immediately preceding example, both EMG and biomechanical modeling showed violin playing to be "low-load". On average, measured EMG remained below 20\% of MVC and, in fact, some muscles consistently used as little as $5 \%$. Such conditions would be considered "safe" for most activities, but are clearly not so for the kinds of muscular (repetitive) use that is needed in the pursuit of artistic excellence in the performing arts. Such work clearly indicates that both researchers and artists need to be willing to re-examine the fundamental beliefs and understandings of their disciplines.

\section{Conclusion}

Even though when compared to sports and kinesiology there is a small body of EMG research investigating arts performance, the examples above illuminate the potential and relevance of EMG applications in this area. Studies based upon skill descriptions and quantification of anatomical \& physiological phenomena will permit better assessments and analyses of technical skills in terms of the training process, artistic development, and the refinement of pedagogical methods - all of which provides foundational information for artists in their pursuit of a long, fulfilling and healthy career. However, artistry is typically an individualistic quest predicated on the strong vision of an outcome. This is a fact that all science-based research needs to carefully consider. As a result, research in the performance arts should be expected to involve a multi-stage process where research design is both 
application driven and can be viewed as relevant by the artistic community. The examples identified in this book chapter show EMG research to have significant application potential in the performing arts; it remains for researchers and artists to engage in the collaborative processes that might encourage more research in this area.

\section{Acknowledgment}

This work was supported by funding of CREDO program/University of Lethbridge and National Sciences and Engineering Research Council of Canada (NSERC).

\section{References}

Albisetti, W., Bartolomeo, O., Gabbiadini, S., Ventura, A., \& Parrini, M. (2008). Surface EMG Evaluation of Patellofemoral Pain Syndrome in a Professional Ballet Dancer. Medical Problems of Performing Artists, 23(1), 29-32.

Bejjani, F., Ferrara, L., Xu, N., Tomaino, C., Pavlidis, L., Wu, J., \& Dommerholt, J. (1989). Comparison of three piano techniques as an implementation of a proposed experimental design. Medical Problems of Performing Artists, 4(3), 109-103.

Chatfield, S. (2003). Variability of Electromyographic and Kinematic Measurement in Dance Medicine and Science Research. Journal of Dance Medicine \& Science, 7(2), 42-48.

Fishbein, M., Middlestadt, S., Ottati, V., Straus, S., \& Ellis, A. (1988). Medical problems among ICSOM musicians:overview of a national survey. Medical Problems of Performing Artists, 3(1), 1-8.

Fry, H. (1986). Overuse syndrome of the upper limb in musicians. Medical Journal of Australia, 144(4), 182-185.

Fry, H. (1988). Patterns of over-use seen in 658 affected instrumental musicians. International Journal of Music Education, 11(1), 3-16.

Heuser, F., \& Mcnittgray, J. L. (1991). Emg Potentials Prior to Tone Commencement in Trumpet Players. Medical Problems of Performing Artists, 6(2), 51-56.

Iltis, P., \& Givens, M. (2005). EMG Characterization of Embouchure Muscle Activity: Reliability and Application to Embouchure Dystonia. [Research]. Medical Problems of Performing Artists, 20(1), 25-34.

Kjelland, J. (2000). Application of Electromyography and Electromyographic Biofeedback in Music Performance Research: A Review of the Literature since 1985. Medical Problems of Performing Artists, 15(3), 115-118.

Middlestadt, S., \& Fishbein, M. (1989). The prevelance of severe musculoskeletal problems among male and female symphony orchestra string players. Medical Problems of Performing Artists, 4(1), 41-48.

Morasky, R. L., Reynolds, C., \& Clarke, G. (1981). Using biofeedback to reduce left arm extensor EMG of string players during musical performance. Biofeedback and selfregulation, 6(4), 565-572.

Riley, k., \& Chong, J. (2010). Garrick Olsson on Technique: mastering the paradoxical element of control. Clavier Companion, 2(5), 12-21.

Shan, G., \& Visentin, P. (2010). Arts Biomechanics - An Infant Science: Its Challenges and Future. NOVA Publishers, ISBN: 978-1-60876-809-7, New York, USA. 
Shan, G., Visentin, P., \& Schultz, A. (2004). Multi-dimensional Signal Analysis as a means of better understanding factors associated with repetitive use in violin performance. Medical Problems of Performing Artists, 19(3), 129-139.

Visentin, P., \& Shan, G. (2003). The kinetic characteristics of the bow arm during violin performance; an examination of internal loads as a function of tempo. Medical Problems of Performing Artists, 18(3), 91-97.

Visentin, P., Shan, G., \& Wasiak, E. B. (2008). Informing music teaching and learning using movement analysis technology. International Journal of Music Education, 26(1), 71-85.

Zaza, C. (1998). Playing-related musculoskeletal disorders in musicians: A systematic review of incidence \& prevalence. Canadian Medical Association Journal, 158(8), 1019-25.

Zinn, M. (1998). The use of sEMG feedback for transfer of relaxation into piano technique [unpublished]. . Master, Illinois Institute of Technology, Chicago. 


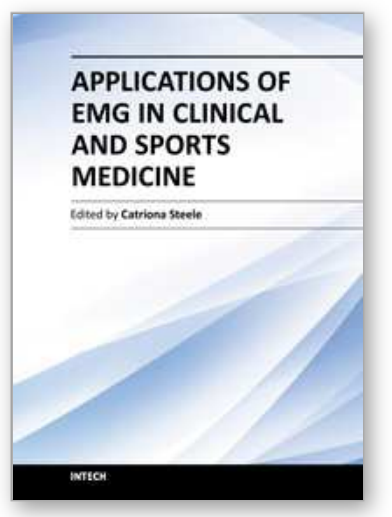

\author{
Applications of EMG in Clinical and Sports Medicine \\ Edited by Dr. Catriona Steele
}

ISBN 978-953-307-798-7

Hard cover, 396 pages

Publisher InTech

Published online 11, January, 2012

Published in print edition January, 2012

This second of two volumes on EMG (Electromyography) covers a wide range of clinical applications, as a complement to the methods discussed in volume 1. Topics range from gait and vibration analysis, through posture and falls prevention, to biofeedback in the treatment of neurologic swallowing impairment. The volume includes sections on back care, sports and performance medicine, gynecology/urology and orofacial function. Authors describe the procedures for their experimental studies with detailed and clear illustrations and references to the literature. The limitations of SEMG measures and methods for careful analysis are discussed. This broad compilation of articles discussing the use of EMG in both clinical and research applications demonstrates the utility of the method as a tool in a wide variety of disciplines and clinical fields.

\title{
How to reference
}

In order to correctly reference this scholarly work, feel free to copy and paste the following:

Gongbing Shan and Peter Visentin (2012). EMG Applications in Studies of Arts, Applications of EMG in Clinical and Sports Medicine, Dr. Catriona Steele (Ed.), ISBN: 978-953-307-798-7, InTech, Available from: http://www.intechopen.com/books/applications-of-emg-in-clinical-and-sports-medicine/emg-applications-instudies-of-arts

\section{INTECH}

open science | open minds

\section{InTech Europe}

University Campus STeP Ri

Slavka Krautzeka 83/A

51000 Rijeka, Croatia

Phone: +385 (51) 770447

Fax: +385 (51) 686166

www.intechopen.com

\section{InTech China}

Unit 405, Office Block, Hotel Equatorial Shanghai

No.65, Yan An Road (West), Shanghai, 200040, China

中国上海市延安西路65号上海国际贵都大饭店办公楼 405 单元

Phone: +86-21-62489820

Fax: +86-21-62489821 
(C) 2012 The Author(s). Licensee IntechOpen. This is an open access article distributed under the terms of the Creative Commons Attribution 3.0 License, which permits unrestricted use, distribution, and reproduction in any medium, provided the original work is properly cited. 